\title{
Perimeter and Area Estimations of Digitized Objects with Fuzzy Borders
}

\author{
Nataša Sladoje $^{1, \star}$, Ingela Nyström ${ }^{1}$, and Punam K. Saha ${ }^{2}$ \\ 1 Centre for Image Analysis, Uppsala, Sweden \\ natasa, ingela\}@cb.uu.se \\ 2 MIPG, Dept. of Radiology, University of Pennsylvania \\ Philadelphia, PA, USA \\ saha@mipg. upenn.edu
}

\begin{abstract}
Fuzzy segmentation methods have been developed in order to reduce the negative effects of the unavoidable loss of data in the digitization process. These methods require the development of new image analysis methods, handling grey-level images. This paper describes the first step in our work on developing shape analysis methods for fuzzy images: the investigation of several measurements on digitized objects with fuzzy borders. The performance of perimeter, area, and the $P^{2} A$ measure estimators for digitized disks and digitized squares with fuzzy borders is analyzed. The method we suggest greatly improves the results obtained from crisp (hard) segmentation, especially in the case of low resolution images.
\end{abstract}

Keywords: Fuzzy shape representation, measurement, accuracy, precision

\section{Introduction}

It has become clear that some of the problems caused by crisp (hard) segmentation of a grey-level image (leading to a binary image) may be solved by using fuzzy segmentation instead (see, e.g., [7]); performing image analysis directly on a grey-level image, i.e., on its corresponding fuzzy segmented image. To date, very little has been published on the development of shape analysis methods that can handle fuzzy segmented images.

We are interested in estimating quantitative properties of fuzzy objects. In particular, we would like to investigate the behaviour of perimeter estimation of a fuzzy object, digitized at relatively low resolution. A comparative evaluation of several estimators of the length of a binary digitized curve is presented in 3]. Generally, they perform asymptotically very well, but produce either overor under-estimation at low resolution. We believe that the perimeter measures computed from a fuzzy segmentation could provide better results. We also expect improved area estimations.

In the context of computing perimeter and area estimators, it is natural to analyze how they affect the $P^{2} A$ shape descriptor, for a shape $S$, calculated as

\footnotetext{
* The first author is supported by a grant from the Swedish Institute.
} 


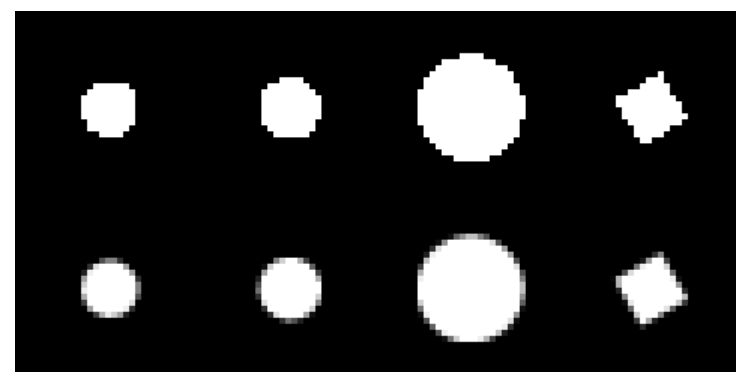

Fig. 1. Examples of digitized objects with crisp (top) and fuzzy border (bottom).

$$
\frac{\operatorname{perimeter}^{2}(S)}{4 \pi \cdot \operatorname{area}(S)}
$$

This is a measure of the compactness of a two-dimensional (2D) object closely related to the well-known isoperimetric inequality,

$$
(\text { perimeter }(S))^{2} \geq 4 \pi \cdot \operatorname{area}(S) .
$$

According to this inequality, for any continuous $2 D$ object, $P^{2} A$ is larger than or equal to 1 . The $P^{2} A$ value of an object decreases with the increase of its compactness and the lower limit is reached for a disk, the most compact object. However, for digitized $2 D$ objects, the $P^{2} A$ measure can be less than 1 , even for non-circular objects. We expect that fuzzification of a digital object increases its similarity to the continuous analogue shape.

\section{Background and Related Works}

We assume that, during a segmentation process, most of the image points can be classified as either object or background, but for some of the points it is hard to make this discrimination. Such points are often located around the border of an object. One adequate way to treat these points may be to determine the extent of their membership to the object/background, i.e., to define an object as a fuzzy subset.

In this study, we compute the membership value of a pixel (a point in a digitized image) as a fraction of its area belonging to the original object, where the discrete analogue of the area is used. Area coverage of a pixel is expressed as the number of subpixels, within the candidate pixel, each having its centroid inside the object. It is achieved by increasing the resolution of the image (i.e., sub-sampling). Examples of digital objects obtained this way, are presented in Figure 1.

As a starting point, we utilize some already-known approaches of measuring the perimeter of a fuzzy subset. We are particularly interested in the results of Rosenfeld [6] and Bogomolny [1. Both papers are related to fuzzy subsets in the 
continuous domain, and utilize the gradient of a fuzzy membership function for the calculation of a perimeter of a set. In this paper, we propose a new gradient estimator for the discrete case.

\subsection{Perimeter of Fuzzy Subsets in the Continuous Domain}

It is natural to define the perimeter of a digital fuzzy subset with respect to the definition of the perimeter of a fuzzy subset in the continuous domain. The notions of area and perimeter of a (continuous) $2 D$ fuzzy subset were introduced in [6], as a generalization of their hard analogues. However, some simple interrelations, e.g., the isoperimetric inequality, that hold in the crisp case, do not hold if the perimeter and area are defined as in [6]. This fact initialized further research and resulted in the modified definition of the perimeter of a fuzzy subset [1].

We are interested in the results related to the fuzzy step subsets which were considered in both [6] and [1]. We utilize the following definitions, cited from [10] (Definition 1) and [1] (Definitions 2-5).

Definition 1 A fuzzy subset $M$ of a reference set $X \in \mathbf{R}^{n}$ is a set of ordered pairs $M=\left\{\left(x, \mu_{M}(x)\right) \mid x \in X\right\}$, where $\mu_{M}: X \rightarrow[0,1]$ is the membership function of $M$ in $X$.

Definition $2 A$ set $S$, given by its membership function $\mu_{S}: \mathbf{R}^{2} \rightarrow[0,1]$, is a fuzzy step subset if

(i) there exist (crisp) open sets $S_{1}, \ldots, S_{n+1}, n=n\left(\mu_{S}\right)$, of which all but one (say, $\left.S_{n+1}\right)$ are bounded;

(ii) $S_{i} \cap S_{j}=\emptyset, \quad i \neq j$;

(iii) $\bigcup_{i=1}^{n+1} \bar{S}_{j}=\mathbf{R}^{2}$, where $\bar{S}$ denotes the closure of a (crisp) set $S$ in the Euclidean topology of $\mathbf{R}^{2}$;

(iv) if $i \neq j$, then $\bar{S}_{i} \cap \bar{S}_{j}=\bigcup_{k=1}^{n_{i j}} B_{i j k}$, where $B_{i j k}$ is a rectifiable Jordan arc of length $l\left(B_{i j k}\right)$;

(v) $\mu_{S}(x)=s_{i}$, for $x \in S_{i}$, i.e., for $i=1,2, \ldots, n+1, S_{i}$ is iso-membership valued, and $s_{n+1}=0$.

Note: A fuzzy digital image is a fuzzy step subset, where sets $S_{i}$ are determined with respect to the connected sets of pixels having the same membership (greylevel) value.

Definition 3 The area $A(M)$ of a fuzzy subset $M$ of a reference set $X$, given by its membership function $\mu_{M}$, is $A(M)=\int_{X} \mu_{M}(x) d x$, where $x \in X$.

Definition 4 (Rosenfeld) The perimeter $P(S)$ of a fuzzy step subset $S$, given by its membership function $\mu_{S}$, is

$$
P(S)=\sum_{\substack{i, j=1 \\ i<j}}^{n+1} \sum_{k=1}^{n_{i j}}\left|s_{i}-s_{j}\right| \cdot l\left(B_{i j k}\right) .
$$


Definition 5 (Bogomolny) The perimeter $P(S)$ of a fuzzy step subset $S$, given by its membership function $\mu_{S}$, is

$$
P(S)=\sum_{\substack{i, j=1 \\ i<j}}^{n+1} \sum_{k=1}^{n_{i j}}\left|\sqrt{s_{i}}-\sqrt{s_{j}}\right| \cdot l\left(B_{i j k}\right) .
$$

For the notification used in Definitions 4 and 5, see Definition 2.

The isoperimetric inequality does not hold if the perimeter is defined by Definition 4, but it holds if Definition 5 is used. Both definitions reduce to the usual definition of a perimeter in the case of a crisp set.

\subsection{Perimeter of Crisp Sets in the Discrete Domain}

The direct application of Definitions 4 and 5 assumes estimation of the length of a border line, $l\left(B_{i j k}\right)$, between two neighbouring sets of pixels, $S_{i}$ and $S_{j}$, having different grey-levels. Consequently, the accuracy of the perimeter estimation of a fuzzy subset strongly depends on the accuracy of the estimation of the length of a line in a binary digitized set.

The results of the comparative evaluation of length estimators, presented in [3], show that the length estimators which are multigrid convergent, i.e., perform very well if the image resolution is high, are all global. It is also noticed that they have disadvantages, if applied to low resolution images. In this paper we focus on the evaluation of estimators at low resolutions. We are not interested in multigrid convergence, but instead use an intuitively simple local approach that relies on measuring elementary moves within the path $l\left(B_{i j k}\right)$, and that is naturally incorporated in the gradient-based method for the perimeter estimation that we suggest.

\section{Perimeter of a Digital Fuzzy Subset}

The perimeter of a digital fuzzy subset is obtained by calculating a measure related to the gradient of the membership function. This measure is determined by summing, for each pixel, the difference between membership values of neighbouring pixels multiplied by the length of the border line between every two neighbouring pixels.

It is assumed that the digital objects we work with are fuzzy (only) on the border; the membership value of the pixels is equal to 1 in the inner - "central" - area of the object, and "radially" decreases toward the border. This intuitively follows from the fuzzification method that we apply and allows us to assume that the objects we work with fulfil the following property:

Definition 6 (Local fuzzy convexity property) A fuzzy digital object has a local fuzzy convexity property if the $3 \times 3$ neighbourhood of each pixel in the image is a convex fuzzy subset. (For the definition of a convex fuzzy subset, see, e.g., [9].) 


$$
\begin{array}{|l|l|l|}
\hline z_{1} & z_{2} & z_{3} \\
\hline z_{4} & z_{5} & z_{6} \\
\hline z_{7} & z_{8} & z_{9} \\
\hline
\end{array}
$$

Fig. 2. The $3 \times 3$ neighbourhood of a pixel $z_{5}$ in a $2 D$ digital image.

Note: A convex fuzzy subset has a local fuzzy convexity property, but the reverse does not hold in general. For each pixel, an increase of membership values in its $3 \times 3$ neighbourhood may occur only in the directions contained in the convex angle, positioned in the observed pixel. Consequently, the estimated gradient in each point of the fuzzy object can be computed in the way described in the sequel.

Let the $3 \times 3$ neighbourhood of a pixel $z_{5}$ be denoted as in Figure 2, To compute the increase of a membership function, i.e., the estimated gradient at $z_{5}$, first, we calculate

$$
\begin{aligned}
d_{\text {hor }} & =\max \left\{\mu\left(z_{6}\right)-\mu\left(z_{5}\right), \mu\left(z_{4}\right)-\mu\left(z_{5}\right), 0\right\}, \\
d_{\text {vert }} & =\max \left\{\mu\left(z_{2}\right)-\mu\left(z_{5}\right), \mu\left(z_{8}\right)-\mu\left(z_{5}\right), 0\right\},
\end{aligned}
$$

and then assign $d_{\max }=\max \left\{d_{\text {hor }}, d_{\text {vert }}\right\}$ and $d_{\min }=\min \left\{d_{\text {hor }}, d_{\text {vert }}\right\}$. The contribution $\operatorname{per}\left(z_{5}\right)$ of the observed pixel $z_{5}$ to the estimated perimeter is determined as

$$
\operatorname{per}\left(z_{5}\right)=d_{\text {min }} \cdot b \text {-step }+\left(d_{\text {max }}-d_{\text {min }}\right) \cdot a \text {-step, }
$$

where $a$-step and $b$-step are the estimates (weights) of the isothetic and the diagonal distance between two neighbouring pixels, respectively, see [2].

The perimeter $P(S)$ of a fuzzy object $S$ in the image $I$ is calculated as

$$
P(S)=\sum_{z \in I} \operatorname{per}(z) .
$$

Locally, equation (1) corresponds to Definition 4. If the square root of the membership value is used instead, the result is in accordance with Definition 5.

To optimize the perimeter estimation of a fuzzy object, methods and results already successfully applied in the binary case are used. For the $a$ - and $b$-step weights, we use the coefficients $a_{n \rightarrow \infty} M S E \approx 0.948$ and $b_{n \rightarrow \infty} M S E \approx 1.343$ $([4],[5])$ to minimize the expected mean square error $(M S E)$ for measurement of the length of long line segments $(n \rightarrow \infty)$ and to give an unbiased estimate. It is shown by Dorst and Smeulders [4] that such a choice of the coefficients provides the best linear unbiased estimator (BLUE for short) for straight lines and that it also performs well when applied to curved lines.

In the binary case, equation (2) reduces to the perimeter estimator where the perimeter corresponds to the outer boundary of an object, which leads to an over-estimation of a real perimeter, especially, for small objects. In that case, the perimeter estimation is highly improved if a (negative) correction term is used. We find that the same holds for fuzzy perimeter estimation. In other words, 
the subtraction of an appropriately chosen constant value from the estimated perimeter compensates for the over-estimation caused by digitization, particularly for small objects. We have determined the correction term CorrTerm as the difference between the mean of the estimated perimeter values of 10,000 (hard) disks of radius 1 pixel, randomly positioned inside a pixel, and the real perimeter value of a disk of the same size. We obtained CorrTerm $=-0.689078$. The perimeter of the fuzzy objects is finally calculated by using the following definition.

Definition 7 The perimeter $P(S)$ of a fuzzy object $S$ is given by the equation

$$
P_{\text {corr }}(S)=\sum_{z \in I} \operatorname{per}(z)-0.689078
$$

where $\operatorname{per}(z)$ is a contribution to the perimeter of any pixel $z$ in the image $I$.

Our choice of correction term, based on the specific shape of a disk, should not be seen as a shape-dependent (however, the possibility of further adjustment of the estimation results for some specific cases should not be excluded). The disk, being the most compact shape, having a smooth boundary, seems to be a reasonable choice to be used for "calibration" of the experimental results.

\section{Results}

We have performed tests for 100 disks for each observed real-valued radius (the centre is randomly positioned inside a pixel) and for 1,000 randomly positioned squares for each observed real-valued side length (for each size 100 random centre positions each with 10 random rotations between 0 and 45 degrees are used). The experiment is repeated for different sub-sample factors (1, 4, 8, and 16). Note that sub-sample factor 1 corresponds to hard segmentation and that it is meaningless to increase the sub-sample factor above 16 for 8-bit pixel values $\left(16 \times 16=256=2^{8}\right)$. We focus on the results obtained for disks with radius up to 20 pixels and for squares with side length up to 30 pixels.

We present results obtained for area, perimeter, and $P^{2} A$ measure estimations. For each of the parameters, we determine the mean of the estimated values, as well as maximal and minimal estimated values.

\subsection{Area Estimation}

The results obtained for area estimation of both digital disks and digital squares with fuzzy border are very encouraging. Even though the number of pixels in the object gives a very good estimate for the area of a (hard) object, the precision of this estimate is rather low for small objects. According to the results presented in Figure 3 for sub-sample factor 1 (corresponding to the binary case), we cannot expect the maximal error to be less than $5 \%$ of the real value, if the radius of the disk is less than 7 pixels. The digitized squares are even more affected by 

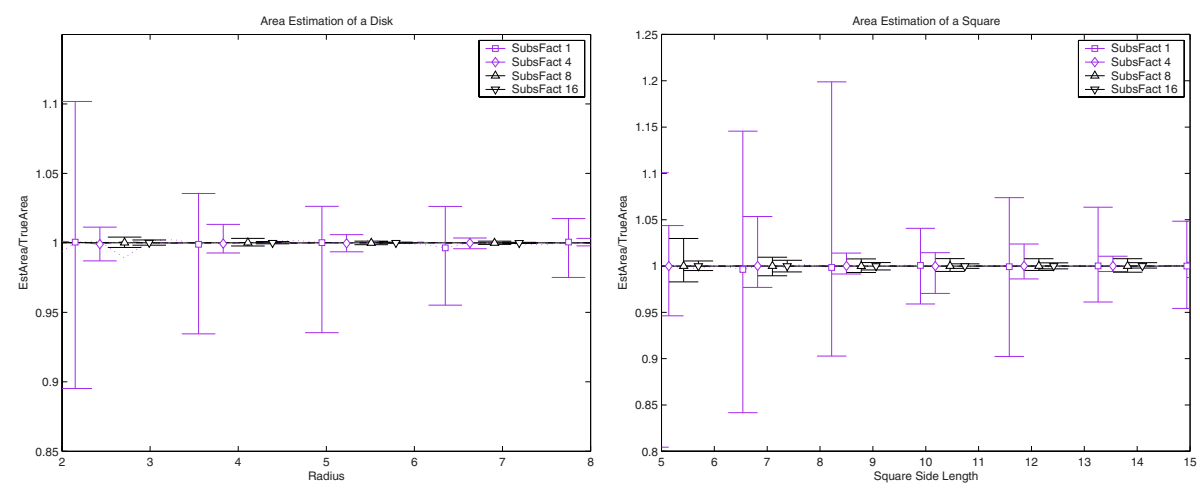

Fig. 3. Area estimation of small size objects randomly positioned in a grid. Results for disks (left) and squares (right).

the problem of imprecise area estimation than the disks, because of the possible appearance of straight line segments aligned with the grid. In that case, theoretically, the order of the area estimation error is equal to the number of points on the straight line segment.

Introducing fuzziness in the segmentation procedure and using the analogous estimate (the sum of pixel values having their centroid within the object, see Definition 3), we get much better results. According to the experiments done for sub-sample factor 8 , the area estimation error is less than $1 \%$ for disks with radius slightly larger than 1 pixel, while the improvement is even more significant in the case of digitized squares, where the error is reduced to less than $1 \%$ for squares with side length 4 pixels. The precision of this unbiased estimation increases both with the size of the object and the sub-sample factor.

\subsection{Perimeter Estimation}

Perimeter estimation of digitized objects is our main topic. The disadvantages of the available estimators are their low precision for small objects and their biased behaviour [3]. We manage to get better estimations by applying fuzzy segmentation both on a disk and a square, and by using the method suggested by Definition 7 both when incorporating Definition 4 and Definition 5 .

In order to test Bogomolny's approach (Definition 5), we used the image where the grey-level in each point is determined by using the square root of the membership of a point in an image, instead of the membership value itself. In this way, (strict) object points and background points are not affected, but the grey-levels of the points on the (fuzzy) border are slightly increased.

Our methods based on Definitions 4 and 5 considerably improve the precision of perimeter estimation of small objects. Results for digitized disks and digitized squares are shown in Figure 4 The error becomes less than $1 \%$ for disks with radius of about 13 pixels, even with sub-sample factor 4 . The estimation is slightly biased (the real perimeter value is over-estimated) if Definition 4 is applied. The results obtained for randomly positioned and randomly rotated 


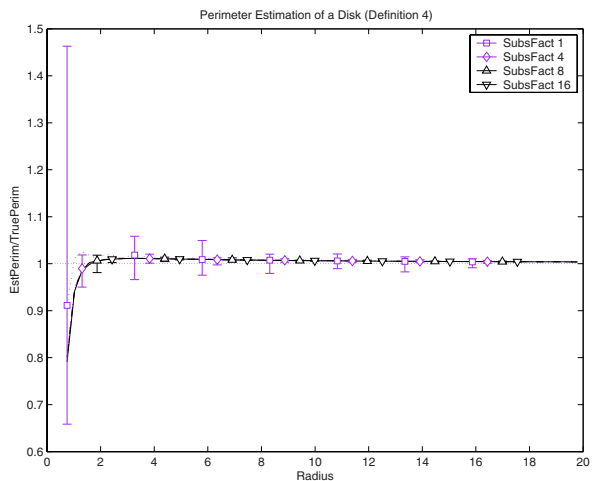

(a)

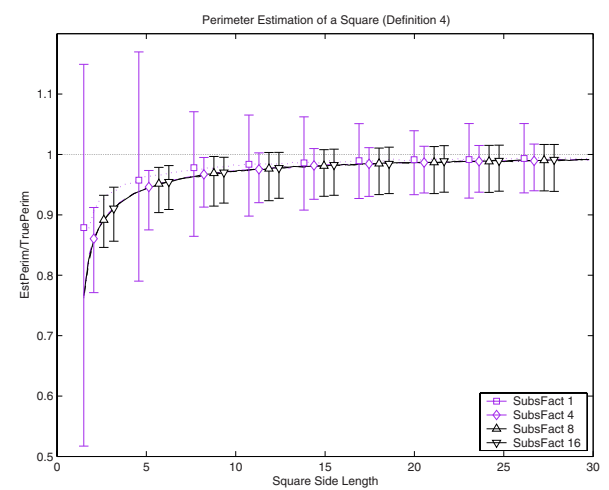

(c)

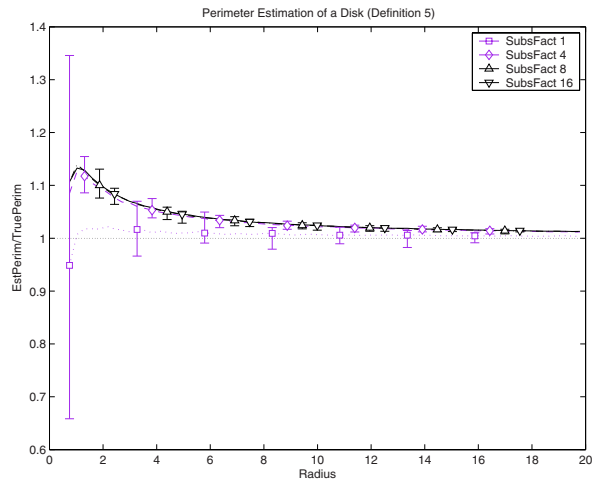

(b)

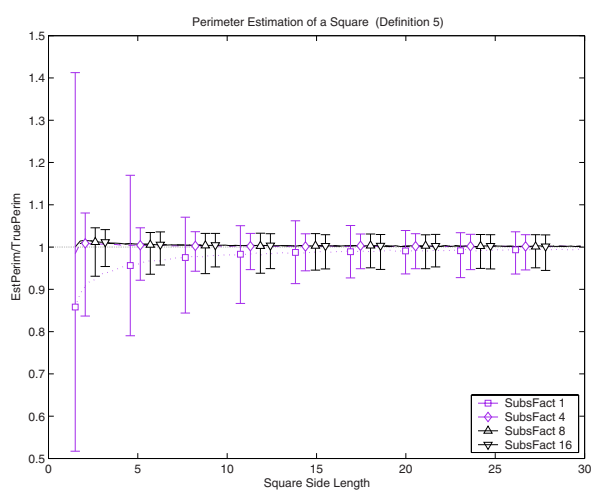

(d)

Fig. 4. Perimeter estimations of disks (top) and squares (bottom) randomly positioned in a grid. (a, c) Definition 4 used. (b, d) Definition 5 used.

squares (the rotation angle is uniformly distributed over the interval $\left[0^{\circ}, 45^{\circ}\right]$ ) of different side lengths are presented in Figure 4(c) and (d).

Our opinion is that estimation method based on Definition 5 is rather nonintuitive, because of the not clearly motivated way of the image transformation (application of the square root function). provide any improvement of the perimeter estimation for the disks; actually, the over-estimation for the disks is much higher compared to the results based on Definition 4, even though the results of a square perimeter estimation are rather good. Consequently, we see no strong reasons to suggest Bogomolny's approach to be used for perimeter estimation. We suggest to select a method expected to be reasonably reliable if applied to general (convex) shapes; according to the results we obtained, our perimeter estimation method based on Definition 4 seems to be a good choice, considerably improving estimation precision even in the very delicate case of a shape bounded by straight line segments aligned with the grid. 

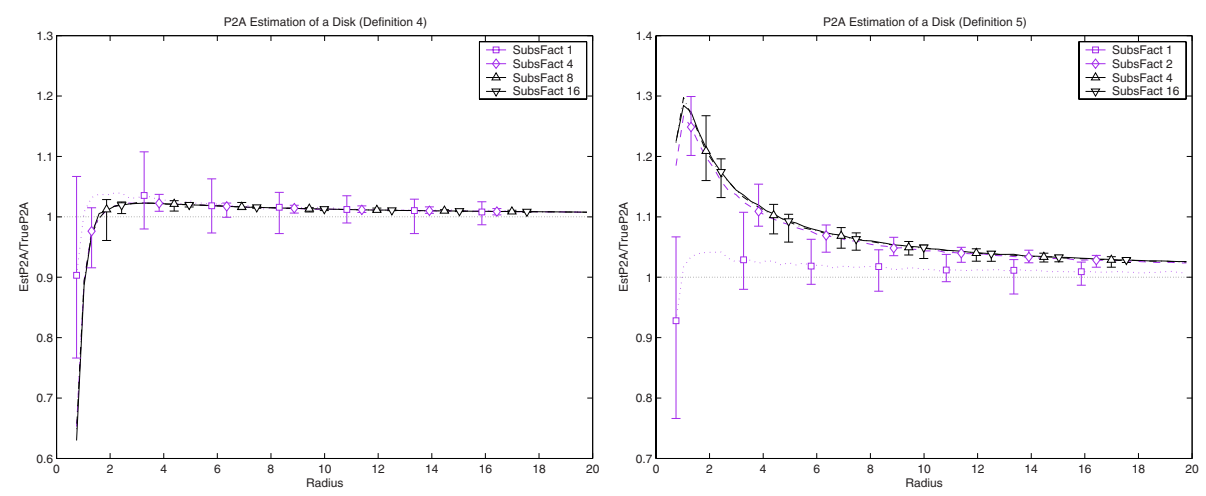

Fig. 5. $P^{2} A$ measure estimation for digitized disks. Left: The results based on Definition 4. Right: The results based on Definition 5 .

\section{3 $\quad P^{2} A$ Measure Estimation}

The results presented in Figure 5 (digitized disks) correspond to $P^{2} A$ measure estimation. We conclude that the estimate for $P^{2} A$ measure for the disks, based on Definition 4, is rather close to the true value (that is 1 for real disks). Not only is the precision of the estimation improved by introducing fuzzy segmentation (i.e., fuzzy border objects), but the $P^{2} A$ measure is larger than 1, i.e., isoperimetric inequality is satisfied for disks with radius slightly larger than 3 pixels for sub-sample factor 4 . This is not the case for hard objects; even though the mean estimated $P^{2} A$ value is above 1 , the estimation is rather imprecise, and results lower than 1 (violating the isoperimetric inequality) may be obtained for disks with radius larger than 20 pixels.

The main intention of Bogomolny's approach is to define perimeter of a (continuous) fuzzy subset in a way that the $P^{2} A$ measure is never less than 1 and is equal to 1 for disks. By using Definition 5 and Definition 7 analogous results should be provided in the discrete case. Obviously, the $P^{2} A$ measure resulting from such a perimeter estimation highly over-estimates the correct value for digital disks, and the over-estimation increases as the sub-sample factor increases. Since the isoperimetric inequality should reduce to equality for disks, we can conclude that the results are even worse than in the binary case.

\section{Comments and Conclusions}

A fuzzy subset representation of a digitized object seems to be promising in improving the precision of estimates of several quantitative properties. The estimation of area, perimeter, and $P^{2} A$ (compactness) measure, for digitized disks and squares, are generally improved when fuzzy membership values are allowed for the pixels instead of just 0 or 1 . The improvements using fuzzy representation have been found to be more significant at low resolution. From the investigation in Section 4.2, we suggest the perimeter estimation method based on Definition 4 
and our Definition 7, as a general and natural one. An important property of this method is that no hard segmentation, which is usually difficult to obtain, is needed; the estimations are based on the fuzzy (border of an) object. This makes our method attractive for applications, where, e.g., the imaging devices produce images with the grey-levels proportional to the picture element coverage, and the grey-levels can be used for defining membership values of a fuzzy object. This is exactly the approach we used in the development of the theoretical background for the shape analysis methods we suggest.

The next step is to study results of applying the proposed method on nonconvex shapes and on shapes with fuzziness appearing not only on the object border. Also, generalizing the method to 3D fuzzy shapes is of interest. The primary goal of this work is to apply the estimation methods on real images where fuzziness of an object is obtained as a result of fuzzy segmentation methods [8].

\section{Acknowledgments}

Prof. Gunilla Borgefors and Dr. Joakim Lindblad, both Centre for Image Analysis, Uppsala, Sweden, are gratefully acknowledged for their scientific support.

\section{References}

1. A. Bogomolny. On the perimeter and area of fuzzy sets. Fuzzy Sets and Systems, 23:257-269, 1987.

2. G. Borgefors. Distance transformations in digital images. Computer Vision, Graphics, and Image Processing, 34:344-371, 1986.

3. D. Coeurjolly and R. Klette. A comparative evaluation of length estimators. In Proceedings of International Conference on Pattern Recognition (ICPR 2002), volume IV, pages 330-334. IEEE Computer Society, August 2002.

4. L. Dorst and A. W. M. Smeulders. Length estimators for digitized contours. Computer Vision, Graphics, and Image Processing, 40:311-333, 1987.

5. Z. Kulpa. Area and perimeter measurement of blobs in discrete binary pictures. Computer Graphics and ImageProcessing, 6:434-454, 1977.

6. A. Rosenfeld and S. Haber. The perimeter of a fuzzy subset. Pattern Recognition, 18:125-130, 1985.

7. P. K. Saha and J. K. Udupa. Relative fuzzy connectedness among multiple objects: Theory, algorithms, and applications in image segmentation. Computer Vision and Image Understanding, 82(1):42-56, Apr. 2001.

8. N. Sladoje, I. Nyström, and P. K. Saha. Measuring perimeter and area in low resolution images using a fuzzy approach. In J. Bigun and T. Gustavsson, eds, Proc. of $13^{\text {th }}$ Scandinavian Conference on Image Analysis (SCIA 2003), Göteborg, Sweden, vol. 2749 of LNCS, pp. 853-860. Springer-Verlag, 2003.

9. X. Yang. Some properties of convex fuzzy sets. Fuzzy Sets and Systems, 72:129-132, 1995.

10. L. Zadeh. Fuzzy sets. Information and Control, 8:338 - 353, 1965. 